\title{
A Comparison of Coordinated TRACE and EIT Observations
}

\author{
M. Zhang \\ Beijing Astronomical Observatory, Chinese Academy of Sciences, \\ China; National Astronomical Observatories, Chinese Academy of \\ Sciences, China.
}

\begin{abstract}
Using coordinated TRACE and EIT observations, we discussed which pair of coronal abundances and ionization equilibrium assumption will give the least difference of temperature and emission maps. It is found that the combination meyer_coronal.abund and shull.ioneq gives the smallest difference between TRACE and EIT observations among the 42 pairs of combination, while the difference between each pair is not much. The difference between temperature or emission maps from coordinated TRACE and EIT observations may be regarded as an estimation of the accuracy of the temperature or emission maps using this kind of method. Under this assumption, the error for temperature diagnosis is estimated at $\triangle\left(\log _{10} T\right)=0.01$ and the error for emission diagnosis is estimated at $\triangle\left(\log _{10} E M\right)=0.08$ at largest.
\end{abstract}

\section{Introduction}

It is well know that in order to get a temperature or an emission map from EUV observations of TRACE or EIT, we have to assume a certain coronal abundances and ionization equilibrium as input. Using different coronal abundances and ionization equilibrium assumption, we will get different temperature and emission maps. This will certainly limit our understanding of the true variation of temperature and emission maps.

Using coordinated TRACE and EIT observations, we will try to discuss which pair of coronal abundances and ionization equilibrium assumption will give the least difference of temperature and emission maps. This pair may be thought as the "best" input for coronal abundances and ionization equilibrium assumption, and the difference between temperature or emission maps from coordinated TRACE and EIT observations may be regarded as an estimation of the accuracy of temperature or emission maps using this kind of method.

\section{Observation and Correlation of TRACE and EIT Observations}

The TRACE maps used here were taken on 01:01:29 17 August 1999 for 171 $\AA$ band and 01:00:45 17 August 1999 for $195 \AA$ band with a pixel size of $0.5^{\prime \prime}$ and a field of view of $8.5^{\prime} \times 8.5^{\prime}$. The full disk EIT maps used here were taken on 01:00:15 17 August 1999 for $171 \AA$ band and 01:13:15 17 August 1999 for 195 

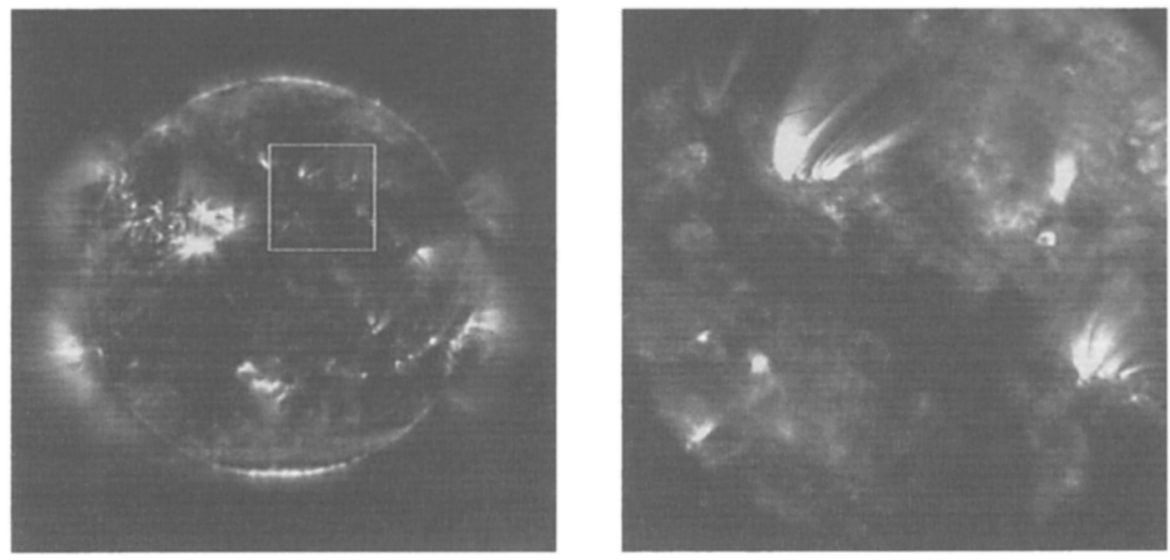

Figure 1. The EIT 171 image (Left) and the TRACE 171 image (Right) analyzed.

$\AA$ band, with a pixel size of $2.6^{\prime \prime}$. Figure 1 shows the EIT 171 image (Left) and the TRACE 171 image (Right) analyzed. For each TRACE image, I smoothed them by $5 \times 5$ pixels and changed the image size to have the same pixel size as EIT's. For each EIT image, I cut a portion that has the same field of view as TRACE image from the EIT full disk image. To have a better correlation with TRACE images, the sub-images from EIT images have also been rotated 0.7 degree.

\section{Compare Different Combination of Coronal Abundances and Ion- ization Equilibrium Assumptions}

The CHIANTI package was used to calculate the synthesis spectra, and the SolarSoft Package was used to calculate the temperature and emission maps. The basic method is to use the ratio between 171 and 195 observations to derive the temperature and emission maps. Figure 2 shows the temperature map derived from EIT observations (Left) and the temperature map derived from TRACE observations (Middle), using coronal abundances assumption from meyer_coronal.abund and ionization equilibrium assumption from shull.ioneq. The difference map of these two is also shown (Right).

Within CHIANTI package, there are seven coronal abundances assumptions (allen.abund, feldman.abund, grevesse_anders.abund, grevesse_sauval98.abund, meyer_coronal.abund, photospheric_may97.abund, waljeski.abund) and six ionization equilibrium assumptions (arnaud_raymond.ioneq, arnaud_raymond_ lmf.ioneq, arnaud_rothenflug.ioneq, arnaud_rothenflug_lmf.ioneq, mazzotta_etal. ioneq, shull.ioneq). This gives $7 \times 6=42$ pairs of combination. 

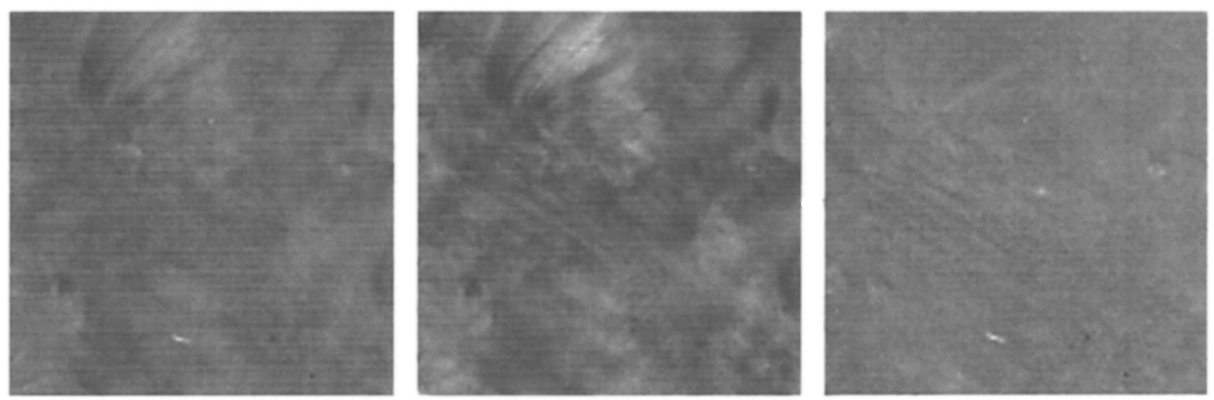

Figure 2. Left: The temperature map derived from EIT data. Middle: The temperature map derived from TRACE data. Right: The difference map.

We compare the difference maps of both temperature and emission maps for all 42 pairs of combination. We found that these pairs give quite similar results each other. It is found that the combination meyer_coronal.abund and shull.ioneq gives the smallest difference between TRACE and EIT observations, while the difference between each pair is not much.

\section{The Accuracy of Temperature and Emission Maps}

Since the temperature and emission maps from TRACE and EIT data are calculated from two independent observations, we may think that the difference between them may be regarded as the (biggest) error for this method (using the ratio between 171 and 195 observations to derive the temperature and emission maps). Under the coronal abundances assumption from meyer_coronal.abund and ionization equilibrium assumption from shull.ioneq, it is found that the (largest) error for temperature diagnosis is $\triangle\left(\log _{10} T\right)=0.01$ and the (largest) error for emission diagnosis is $\triangle\left(\log _{10} E M\right)=0.08$.

It should be mentioned that this error is not caused by the 'temperature resolution' when calculating spectrums with CHIANTI. We have test different 'temperature resolution' spectrums (from $\triangle\left(\log _{10} T\right)=0.001$ to $\triangle\left(\log _{10} T\right)=$ $0.005)$, they all give almost the same results.

\section{Summary}

By comparing temperature and emission maps derived from coordinated TRACE and EIT observations, it is found that the combination meyer_coronal.abund and shull.ioneq gives the smallest difference among the 42 pairs of combination, while the difference between each pair is not much. It is also found that the (largest) error for temperature diagnosis is estimated at $\triangle\left(\log _{10} T\right)=0.01$ and the (largest) error for emission diagnosis is estimated at $\triangle\left(\log _{10} E M\right)=0.08$. 